\title{
eHealth and mHealth Psychosocial Interventions for Youths With Chronic Illnesses: Systematic Review
}

Nancy Lau ${ }^{1,2}$, PhD; Shayna Waldbaum ${ }^{3}$, BSc; Ryan Parigoris ${ }^{4}$, BA; Alison O'Daffer ${ }^{1}$, BA; Casey Walsh ${ }^{5,6}$, MSW, $\mathrm{PhD}$; Susannah F Colt ${ }^{7}$, MA; Joyce P Yi-Frazier ${ }^{1}$, PhD; Tonya M Palermo ${ }^{8,9}$, PhD; Elizabeth McCauley ${ }^{2,9}, \mathrm{PhD}$; Abby R Rosenberg $^{1,10,11}$, MA, MSc, MD

${ }_{1}^{1}$ Palliative Care and Resilience Lab, Center for Clinical and Translational Research, Seattle Children's Research Institute, Seattle, WA, United States

${ }^{2}$ Department of Psychiatry and Behavioral Sciences, University of Washington School of Medicine, Seattle, WA, United States

${ }^{3}$ Chicago Medical School, Rosalind Franklin University of Medicine and Science, North Chicago, IL, United States

${ }^{4}$ Department of Psychology, University of Massachusetts Boston, Boston, MA, United States

${ }^{5}$ Department of Health Services, University of Washington, Seattle, WA, United States

${ }^{6}$ Clinical Research Division, Fred Hutchinson Cancer Research Center, Seattle, WA, United States

${ }^{7}$ Perelman School of Medicine, University of Pennsylvania, Philadelphia, PA, United States

${ }^{8}$ Department of Anesthesiology and Pain Medicine, University of Washington School of Medicine, Seattle, WA, United States

${ }^{9}$ Center for Child Health, Behavior, and Development, Seattle Children's Research Institute, Seattle, WA, United States

${ }^{10}$ Cambia Palliative Care Center of Excellence, University of Washington, Seattle, WA, United States

${ }^{11}$ Department of Pediatrics, University of Washington School of Medicine, Seattle, WA, United States

\section{Corresponding Author:}

Nancy Lau, PhD

Palliative Care and Resilience Lab

Center for Clinical and Translational Research

Seattle Children's Research Institute

1920 Terry Ave

Seattle, WA, 98101

United States

Phone: 12068840569

Email: nancy.lau@ seattlechildrens.org

\section{Abstract}

Background: An estimated $12.8 \%$ of children and adolescents experience chronic health conditions that lead to poor quality of life, adjustment and coping issues, and concurrent mental health problems. Digital health deployment of psychosocial interventions to support youth with chronic illness has become increasingly popular with the advent of the technological advances in the digital age.

Objective: Our objectives were to systematically review published efficacy studies of eHealth and mHealth (mobile health) psychosocial interventions for youths with chronic illnesses and review intervention theory and treatment components.

Methods: PubMed, Embase, Web of Science, PsycInfo, and Cochrane Database of Systematic Reviews were searched for studies published from 2008 to 2019 of eHealth and mHealth psychosocial interventions designed for children and adolescents with chronic illnesses in which efficacy outcomes were reported. We excluded studies of interventions for caregivers, healthy youth, disease and medication management, and telehealth interventions that function solely as a platform to connect patients to providers via phone, text, or videoconference.

Results: We screened 2551 articles and 133 relevant full-text articles. Sixteen efficacy studies with psychosocial and health outcomes representing 12 unique interventions met the inclusion criteria. Of the included studies, 12 were randomized controlled trials and 4 were prospective cohort studies with no comparison group. Most interventions were based in cognitive behavioral theory and designed as eHealth interventions; only 2 were designed as mHealth interventions. All but 2 interventions provided access to support staff via text, phone, email, or discussion forums. The significant heterogeneity in intervention content, intervention structure, medical diagnoses, and outcomes precluded meta-analysis. For example, measurement time points ranged from immediately postcompletion of the mHealth program to 18 months later, and we identified 39 unique outcomes of interest. The 
majority of included studies $(11 / 16,69 \%)$ reported significant changes in measured health and/or psychosocial posttreatment outcomes, with small to large effect sizes.

Conclusions: Although the available literature on the efficacy of eHealth and mHealth psychosocial interventions for youth with chronic illnesses is limited, preliminary research suggests some evidence of positive treatment responses. Future studies should continue to evaluate whether digital health platforms may be a viable alternative model of delivery to traditional face-to-face approaches.

(JMIR Pediatr Parent 2020;3(2):e22329) doi: 10.2196/22329

\section{KEYWORDS}

pediatrics; chronic illness; mHealth; eHealth; psychosocial interventions; mental health

\section{Introduction}

An increasing number of youths (ie, children and adolescents aged 18 years and younger) are diagnosed with a chronic condition in the United States, with an estimated prevalence rate of $12.8 \%$ [1-3]. Chronic illness in childhood negatively impacts quality of life [4,5]. Chronic health conditions can lead to emotional challenges and heighten coping difficulties [6]. Up to $60 \%$ of children with a chronic illness have at least one co-occurring psychological disorder [7], compared with $10 \%$ to $20 \%$ of the general pediatric population [8]. Across illness type, stressors associated with chronic disease are vastly similar. Burdens to these populations include treatment-related stress, changes to daily life and routines, and uncertainty about the future [7,9].

Face-to-face psychosocial interventions such as cognitive behavioral therapy are designed to teach and bolster coping skills and improve psychological adjustment [10]. Such interventions have been developed for various illness populations to improve psychosocial outcomes and quality of life [11]. However, barriers to in-person treatment include limited availability of and access to psychosocial clinicians and high costs of treatment $[12,13]$. A majority of youths screened in school and primary care settings with elevated mental health symptoms do not follow-up with referrals to mental health clinicians, especially those who are racial/ethnic minorities, have public insurance, or come from low-income households [14]. Additionally, there are workforce shortages in proportion to demand and need, with wait times for psychiatric care appointments exceeding that of pediatricians [15]. A potential solution is leveraging technological advances and digital media to deploy behavioral health interventions on a larger scale. Internet-based interventions (otherwise known as eHealth interventions) confer the advantages of instant availability, anonymity, self-pacing, the ability to reach patients in remote areas, and cost-effectiveness due to reduced personnel and infrastructure requirements $[16,17]$.

Internet-based interventions may be particularly appealing to younger generations who are digital natives accustomed to interacting on smartphones and the internet [16-18]. The internet serves as a primary means of health-related and mental health-related information-seeking and communication for youths $[19,20]$. Additionally, young people endorse reluctance to seek psychological services due to social stigma, discomfort discussing personal problems, and a preference for self-help $[16,17,19,21]$. Thus, there has been a rapid growth in the use of eHealth platforms to deploy skills-based behavioral health programs for youths. Moreover, in recent years with the increased use of smartphones there has been a corresponding increase in mobile health (mHealth) apps for symptom self-management on smartphone devices [22,23].

Previous systematic reviews have examined digital interventions in pediatric populations for disease self-management and alleviation of mental health symptoms. Examples include remote management of pediatric chronic pain [24] and technological interventions for asthma self-management in children and adolescents [25-27]. Other reviews have focused on digital health interventions for youth mental health problems [28,29] and internet-based cognitive behavioral therapy for children and adolescents [17,30]. The literature has addressed the benefits of eHealth interventions for anxiety and depression [31], technological tools for disease self-management [32], and technology-based family interventions for improving family functioning [33].

To our knowledge, no existing systematic review has been conducted to critically review the literature on eHealth and mHealth psychosocial interventions for youths with chronic illnesses. Previous reviews in this topic area have focused more narrowly on specific chronic conditions, constellations of mental health symptoms, or types of psychotherapy. Our study objectives were to systematically review the efficacy of eHealth and mHealth psychosocial interventions for youths with chronic illnesses and review underlying intervention theory and treatment components.

\section{Methods}

\section{Literature Search}

The search was executed by a research librarian in five databases for articles published from 2008 to 2019: PubMed/MEDLINE, Embase, Web of Science, PsycINFO, and Cochrane Database of Systematic Reviews. We used keywords and Boolean operators [34] to identify original articles on eHealth and mHealth psychosocial interventions designed for youths or young adults with chronic illnesses. Inclusion criteria were (1) available in English; (2) published in peer-reviewed journal; (3) experimental, quasi-experimental, and observational studies in which efficacy outcome(s) were reported; (4) eHealth or mHealth psychosocial interventions (with technology as the primary mode of content delivery, either entirely self-guided or human-assisted); and (5) designed for children and 
adolescents aged 18 years and younger with chronic disease (ie, a long-term medical condition lasting 3 months or longer [35]).

The original primary search strategy with generic chronic illness search terms is shown in Multimedia Appendix 1. Cancer is a specific condition of interest for our research group for which we were aware of existing digital intervention literature. Based on our primary search strategy, the authors identified several known published studies on digital interventions related to 10 specific chronic illnesses. To ensure all relevant articles were captured, a supplemental search strategy related to the specific chronic illnesses identified was then conducted to generate systematic reviews to search some of the more advanced digital intervention science in pediatrics (Multimedia Appendix 2). Our search strategy was guided and conducted by a medical librarian with extensive experience with systematic reviews; the list of search terms for specific chronic illnesses was modeled after published Cochrane reviews (eg, Law et al [36] on caregiver interventions for children with chronic illness). We excluded studies of interventions that target caregivers or health care providers only, interventions that target mental health problems/disorders not in the context of a chronic medical condition, prevention programs for healthy controls, disease and medication management programs, and programs in which the telehealth platform is only used to connect patients to providers via phone, text, or videoconference.

\section{Selection of Studies}

First, we screened titles and abstracts of studies retrieved for inclusion and exclusion. We then obtained full texts of articles designated as potentially meeting inclusion criteria to assess for eligibility. Screening of all titles, abstracts, and full-text articles was first independently double-coded by authors in pairs (NL, SW; NL, RP; NL, SFC); each dyad coded a subset of articles and NL coded all articles. Then, disagreements between the authors in each dyad were resolved through discussion while referencing the original source material to reach consensus. Finally, for articles meeting inclusion criteria, we independently double-coded relevant information from each study in pairs (NL, SW; NL, RP; NL, SFC), including study design, sample size, target illness, intervention characteristics (eg, intervention theory and components, eHealth or mHealth platform), and treatment outcomes data.

For intervention characteristics, we relied on authors' descriptions, either provided in the articles themselves or in prior publications of the intervention referenced in the included articles, and standard norms for psychosocial interventions. For example, if authors described an intervention as being based on cognitive behavioral theory, we coded the theory as cognitive behavioral; if the intervention followed a prespecified order mirroring the stepwise progression of traditional manualized evidence-based psychotherapies, it was coded as a modular treatment session per clinical norms [37]; and if patients could connect with research or psychosocial staff for support, we coded the intervention as human-assisted.

Unsurprisingly, measures collected in studies incorporated both psychosocial outcomes and physical health/disease-related outcomes. In the context of chronic medical conditions, physical and psychological consequences are intertwined and physical health/disease-related outcomes tend to improve alongside emotional and psychological functioning [38]. Outcome measures were categorized as either psychosocial (depression, social problem solving, fear and worry about symptoms, anxiety sensitivity, perceived stress, rewarding pain behavior, quality of life, social acceptance, family conflict, pain catastrophizing, psychological well-being, emotional functioning, parental protectiveness, anxiety, school attendance, self-efficacy, posttraumatic stress symptoms, somatic symptoms, coping strategies) or physical health/disease-related (fatigue, physical functioning, energy, disease symptoms, pain intensity and frequency, pain interference, pain reactivity, sleep, disease knowledge, activity limitations, functional disability). Categorizations were based on the psychosocial background literature [39-41] and agreed upon internally by our interdisciplinary research team which includes intervention science researchers, health services researchers, physicians, and psychologists; we acknowledge that some outcomes such as aspects of pain management, sleep, and functional impairments may fit either categorization. For study design, we used author designations. For example, a study was categorized as a pilot randomized controlled trial (RCT) if described as such in the article. We referred to the articles to resolve any discrepancies during consensus conversations and did not make inferences beyond authors' definitions and descriptions.

After review of the articles, the team determined that heterogeneity in outcome variables and measurement time points precluded meta-analysis. Thus, we described the data systematically.

\section{Quality Assessment}

We independently assessed study quality in pairs (NL, SW; NL, RP; NL, SFC) using the Cochrane Collaboration's tool for assessing risk of bias [42] to evaluate random sequence generation (selection bias), allocation concealment (selection bias), blinding of participants and personnel (performance bias), blinding of outcome assessment (detection bias), incomplete outcome data (attrition bias), selective reporting (reporting bias), and other biases. We coded each category as low, high, or unclear risk of bias according to established standards in the Cochrane handbook for systematic reviews of interventions [43]. We resolved discrepancies in coding during regularly scheduled consensus meetings by referring to the journal articles themselves.

\section{Results}

\section{Literature Search}

The results of the search and selection of studies are described in the preferred reporting items for systematic reviews and meta-analyses (PRISMA) flow diagram (Figure 1). We screened 2551 articles; 2418 were initially excluded because they did not meet selection criteria. Evaluation of the remaining 133 relevant full-text articles resulted in the exclusion of 117 articles, leaving us with 16 articles that met criteria for inclusion. We provide a synthesis of the findings from the included studies structured around the type of intervention, target population characteristics, intervention content, and type of outcomes. 
Figure 1. Preferred reporting items for systematic reviews and meta-analyses diagram.

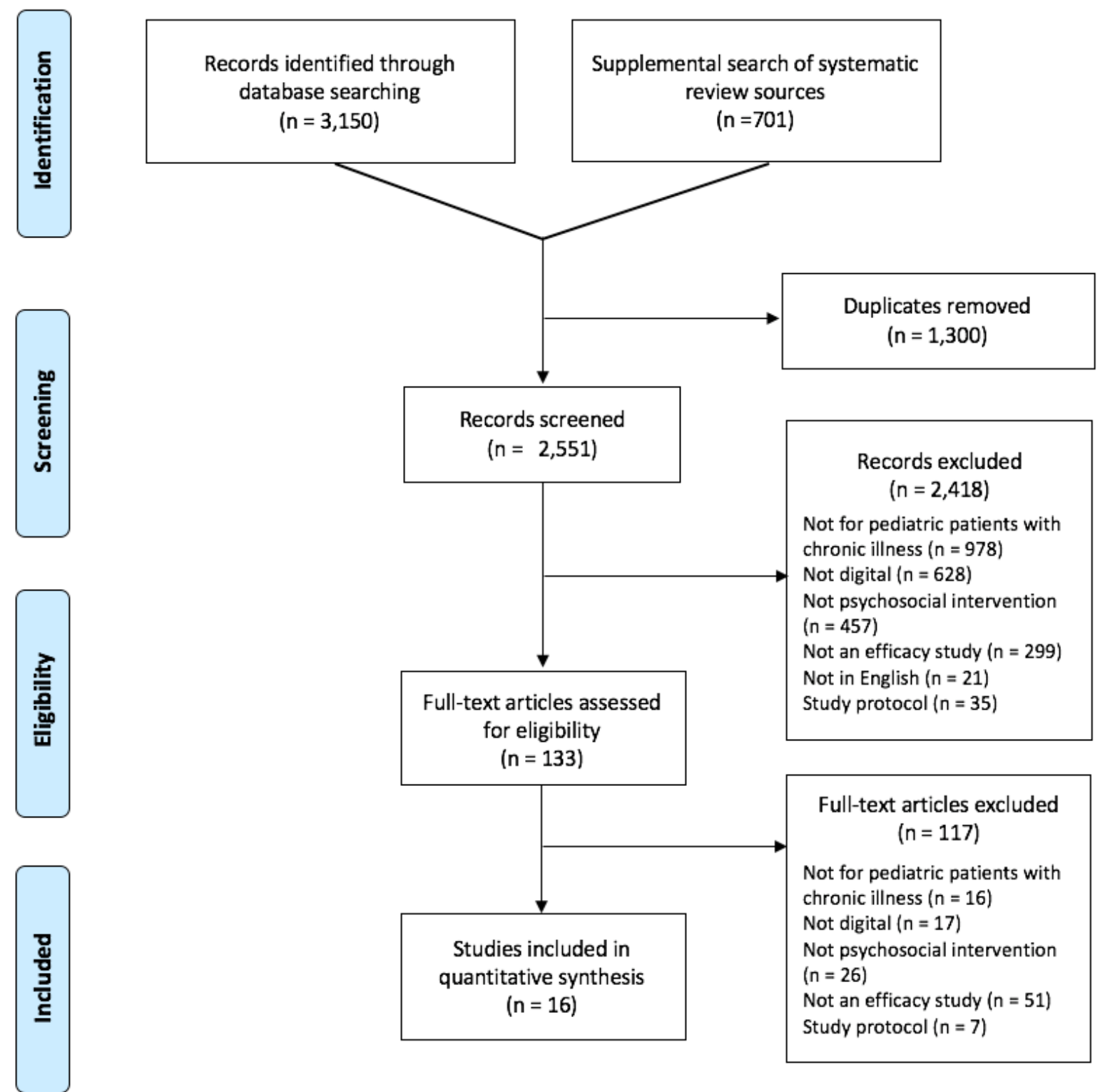

\section{Intervention Characteristics}

We found 12 [44-60] unique digital interventions that were developed and tested in the United States [51,55,58], Australia [44], Canada [49,60], Germany [48,54], the Netherlands [47], and Sweden [45,61] (Table 1). Ten were eHealth interventions [44-48,51-59,61] and 2 mHealth interventions [49,60]. We found that over half $(7 / 12,58 \%)[45-48,53-58,61]$ were based on cognitive behavioral therapy principles [62,63]. Other treatment frameworks represented included disease self-management, problem solving, psychoeducation, and social-emotional learning. The interventions varied in length, duration, and number of sessions. All but two interventions incorporated modular treatment sessions that follow a prespecified order (Table 1). 
Table 1. Interventions targeted for youth chronic illness populations.

\begin{tabular}{|c|c|c|c|c|c|c|c|c|}
\hline Intervention name & $\begin{array}{l}\text { Targeted } \\
\text { illness }\end{array}$ & $\begin{array}{l}\text { Age } \\
\text { range }^{\text {a }}\end{array}$ & $\begin{array}{l}\text { Country } \\
\text { of origin }\end{array}$ & Platform & $\begin{array}{l}\text { Standalone } \\
\text { intervention or } \\
\text { supplement }\end{array}$ & $\begin{array}{l}\text { Iterative } \\
\text { design } \\
\text { process }\end{array}$ & $\begin{array}{l}\text { Modular or } \\
\text { unstructured } \\
\text { sessions }\end{array}$ & Intervention theory \\
\hline $\begin{array}{l}\text { Breathe Easier On- } \\
\text { line [44] }\end{array}$ & $\begin{array}{l}\text { Chronic res- } \\
\text { piratory con- } \\
\text { dition }\end{array}$ & $10-17$ & Australia & eHealth & Standalone & & Modular & $\begin{array}{l}\text { Evidence-based social sup- } \\
\text { port and problem-solving } \\
\text { program following PACE } \\
\text { principle }\end{array}$ \\
\hline $\begin{array}{l}\text { Fatigue in Teenagers } \\
\text { on the InterNET } \\
{[45]}\end{array}$ & $\begin{array}{l}\text { Chronic fa- } \\
\text { tigue syn- } \\
\text { drome }\end{array}$ & $12-18$ & $\begin{array}{l}\text { Nether- } \\
\text { lands }\end{array}$ & eHealth & Standalone & & Modular & Evidence-based $\mathrm{CBT}^{\mathrm{C}}$ \\
\hline iCanCope [60] & Chronic pain & $15-18$ & Canada & mHealth & Standalone & $\mathrm{x}$ & Unstructured & $\begin{array}{l}\text { Evidence-based psychologi- } \\
\text { cal pain management, } \\
\text { symptom monitoring }\end{array}$ \\
\hline $\begin{array}{l}\text { iCBT }^{d} \text { for adoles- } \\
\text { cents with FGID } \\
{[46,61]}\end{array}$ & $\begin{array}{l}\text { Pain-promi- } \\
\text { nent FGID }\end{array}$ & $13-17$ & Sweden & eHealth & Standalone & $\mathrm{x}$ & Modular & Evidence-based iCBT \\
\hline Move It Now [47] & Chronic pain & $12-17$ & $\begin{array}{l}\text { Nether- } \\
\text { lands }\end{array}$ & eHealth & Standalone & & Modular & Evidence-based CBT \\
\hline Onco-STEP [48] & $\begin{array}{l}\text { Survivors of } \\
\text { pediatric } \\
\text { cancer }\end{array}$ & $\geq 15$ & Germany & eHealth & Standalone & & Modular & Evidence-based CBT \\
\hline PainSquad+ $[49,50]$ & $\begin{array}{l}\text { Cancer pain } \\
\text { management }\end{array}$ & $12-18$ & Canada & mHealth & Standalone & $\mathrm{x}$ & Unstructured & $\begin{array}{l}\text { Evidence-based pharmaco- } \\
\text { logical and psychological } \\
\text { pain management strategies }\end{array}$ \\
\hline TeenCope [51] & $\begin{array}{l}\text { Type } 1 \text { dia- } \\
\text { betes }\end{array}$ & $11-14$ & US & eHealth & Standalone & $\mathrm{x}$ & Modular & $\begin{array}{l}\text { Evidence-based psychoedu- } \\
\text { cational intervention based } \\
\text { on coping skills training }\end{array}$ \\
\hline $\begin{array}{l}\text { Teens Taking } \\
\text { Charge: Managing } \\
\text { Arthritis Online [52] }\end{array}$ & $\begin{array}{l}\text { Juvenile idio- } \\
\text { pathic arthri- } \\
\text { tis }\end{array}$ & $12-18$ & Canada & eHealth & Standalone & $\mathrm{x}$ & Modular & $\begin{array}{l}\text { Evidence-based self-manage- } \\
\text { ment strategies }\end{array}$ \\
\hline $\begin{array}{l}\text { Trautmann self-help } \\
\text { programs: internet- } \\
\text { based CBT, internet- } \\
\text { based applied relax- } \\
\text { ation }[53,54]\end{array}$ & $\begin{array}{l}\text { Recurrent } \\
\text { headache }\end{array}$ & $10-18^{\mathrm{a}}$ & Germany & eHealth & Standalone & $\mathrm{x}$ & Modular & $\begin{array}{l}\text { Evidence-based CBT, relax- } \\
\text { ation }\end{array}$ \\
\hline $\begin{array}{l}\text { Web-based manage- } \\
\text { ment of adolescent } \\
\text { pain [55-57] }\end{array}$ & $\begin{array}{l}\text { Chronic } \\
\text { pain, recur- } \\
\text { rent } \\
\text { headache, } \\
\text { sickle cell } \\
\text { disease }\end{array}$ & $11-18^{\mathrm{a}}$ & US & eHealth & Standalone & $\mathrm{x}$ & Modular & Evidence-based CBT \\
\hline $\begin{array}{l}\text { Web-based treat- } \\
\text { ment for adolescents } \\
\text { with } \operatorname{IBD}^{\mathrm{f}}[58]\end{array}$ & $\begin{array}{l}\text { Inflammato- } \\
\text { ry bowel dis- } \\
\text { ease }\end{array}$ & $12-17$ & US & eHealth & $\begin{array}{l}\text { Supplement to } \\
\text { face-to-face }\end{array}$ & $\mathrm{x}$ & Modular & Evidence-based CBT \\
\hline
\end{tabular}

${ }^{a}$ Age range represents lowest and highest age range across all studies for the same intervention.

bACE: problem identification, alternative solution generation, consequences of each alternative solution, execute solution and evaluate.

${ }^{\mathrm{c}} \mathrm{CBT}$ : cognitive behavioral therapy.

$\mathrm{d}_{\mathrm{iCBT}}$ : internet-based cognitive behavioral therapy.

${ }^{\mathrm{e}}$ FGID: functional gastrointestinal disorder.

fIBD: inflammatory bowel disease.

All except two were human-assisted (10/12, 83\%); human-assisted interventions allowed patients to connect with research or psychosocial staff (ie, psychologist, psychology trainee, nurse, peer counselor) for support via emails, texts, phone calls, private online messaging centers, or message boards
(Multimedia Appendix 3). Half of the interventions $(6 / 12,50 \%)$ had some element of caregiver involvement [45-47,52,55-58,61]. Skills practice was notably the only component shared across all interventions. Other components incorporated that lend themselves well to digital intervention platforms include 
ecological momentary assessment, which allows symptom tracking in real time $(4 / 12,33 \%)$ [64]; tailoring of content to individual users $(6 / 12,50 \%)$; didactic videos $(4 / 12,33 \%)$; online discussion forums $(6 / 12,50 \%)$; and gamification to increase engagement $(3 / 12,25 \%)$.

\section{Participants and Study Characteristics}

Participant ages ranged from 10 to 18 years. The targeted chronic illnesses included diabetes, chronic pain, juvenile arthritis, cancer, chronic fatigue syndrome, recurrent headache, chronic respiratory condition, sickle cell disease, and gastrointestinal disorders (Table 1).

Of the 16 included efficacy studies (Tables 2 and 3), there were 3 single-site prospective cohort studies [44,53,57], 1 multisite prospective cohort study [52], 5 pilot RCTs (3 single-site [46,48,58], 2 multisite $[49,60])$, and 7 phase 2-3 RCTs (4 single-site $[45,46,54,56,61], 3$ multisite [47,55,59]). The 4 non-RCT prospective cohort studies were pilot studies with small sample sizes ranging from 2061 to 4049 participants with no comparison groups. For the pilot RCTs, sample sizes ranged from 1853 to 8357; one was described as powered for between-groups analyses [57]. The majority had an active treatment comparison condition, with only one using a wait-list control [56]. For the RCTs, sample sizes ranged from 4856 to 32,059; five were described as powered for between-groups analyses [45,55,56,59,61]. A little over half (4/7, 57\%) $[45,53-55,57,59,61]$ were compared with an active treatment condition, and the rest were compared with a wait-list control group.

Measures of psychosocial outcomes were collected across all studies, and physical health outcomes were collected in $88 \%$ (14/16) of studies [45-47,49,52-61] (Tables 2 and 3). However, outcomes assessed were heterogeneous, which prevents holistic synthesis across studies. At posttreatment, 56\% (9/16) of the reviewed studies reported significant improvements in psychosocial outcomes (eg, anxiety, depression) $[46,48,49,55,58,61]$; effect sizes, where reported, ranged from small to large for RCTs and non-RCTs alike [46-49,55,58,61]. At posttreatment, half $(8 / 16,50 \%)[45-47,49,52,53,56]$ of the reviewed studies reported significant improvements in health-related outcomes (eg, physical functioning, disease knowledge); effect sizes, where reported, ranged from small to large for RCTs and non-RCTs alike [46,47,49,52,55,56,61]. In combination, a majority $(11 / 16,69 \%)$ of included studies reported some evidence of efficacy on psychosocial outcomes and/or health-related outcomes at posttreatment [45-49,52,53,55,56,58,59]. Findings across RCTs and non-RCTs were similar, with the exception that all non-RCTs reported some improvements in psychosocial outcomes. 
Table 2. Original research publications with quantitative outcomes: randomized controlled trials only.

\begin{tabular}{|c|c|c|c|c|c|c|}
\hline $\begin{array}{l}\text { Intervention } \\
\text { name }\end{array}$ & Type of study & Control group & $\begin{array}{l}\text { Sample } \\
\text { size }\end{array}$ & $\begin{array}{l}\text { Powered } \\
\text { for } \\
\text { analyses? }\end{array}$ & Posttreatment outcomes ${ }^{\mathrm{a} \cdot \mathrm{b}}$ & Longitudinal outcomes $^{\mathrm{a} \cdot \mathrm{b}}$ \\
\hline $\begin{array}{l}\text { Breathe Easier } \\
\text { Online [44] }\end{array}$ & Pilot $\mathrm{RCT}^{\mathrm{c}}$ & Wait-list control & 42 & & $\begin{array}{l}\text { Psychosocial outcomes: no sig- } \\
\text { nificant results for depression } \\
\text { or social problem solving }\end{array}$ & $N / A^{d}$ \\
\hline \multirow[t]{2}{*}{$\begin{array}{l}\text { Fatigue in } \\
\text { Teenagers on the } \\
\text { InterNET [45] }\end{array}$} & \multirow[t]{2}{*}{$\mathrm{RCT}$} & \multirow[t]{2}{*}{ Usual care } & \multirow[t]{2}{*}{135} & \multirow[t]{2}{*}{$\mathrm{x}$} & $\begin{array}{l}\text { Psychosocial outcomes: inter- } \\
\text { vention improved school atten- } \\
\text { dance ( } P \leq .01,95 \% \text { CI } 2.7 \text { to } \\
8.9)\end{array}$ & $\begin{array}{l}\text { Psychosocial outcomes: } \\
\text { intervention improved } \\
\text { school attendance at } 12 \\
\text { months }\end{array}$ \\
\hline & & & & & $\begin{array}{l}\text { Physical health outcomes: inter- } \\
\text { vention improved fatigue } \\
(P \leq .01,95 \% \text { CI } 2.1 \text { to } 4.9) \text { and } \\
\text { physical functioning }(P \leq .01 \text {, } \\
95 \% \text { CI } 2.3 \text { to } 6.3)\end{array}$ & $\begin{array}{l}\text { Physical health outcomes: } \\
\text { intervention improved fa- } \\
\text { tigue and physical function- } \\
\text { ing at } 12 \text { months }\end{array}$ \\
\hline \multirow[t]{2}{*}{ iCanCope [60] } & \multirow[t]{2}{*}{$\begin{array}{l}\text { Pilot multisite } \\
\text { RCT }\end{array}$} & \multirow{2}{*}{$\begin{array}{l}\text { iCanCope version A } \\
\text { (symptom tracking } \\
\text { only) }\end{array}$} & \multirow[t]{2}{*}{59} & & $\begin{array}{l}\text { Psychosocial outcomes: no sig- } \\
\text { nificant results for mood }\end{array}$ & \multirow[t]{2}{*}{ N/A } \\
\hline & & & & & $\begin{array}{l}\text { Physical health outcomes: no } \\
\text { significant results for pain inten- } \\
\text { sity and interference, physical } \\
\text { activity, sleep quality, or ener- } \\
\text { gy }\end{array}$ & \\
\hline \multirow[t]{2}{*}{$\begin{array}{l}\text { ICBT }^{\mathrm{e}} \text { for adoles- } \\
\text { cents with FGID } \\
\text { [61] }\end{array}$} & \multirow[t]{2}{*}{$\mathrm{RCT}$} & \multirow[t]{2}{*}{ Wait-list control } & \multirow[t]{2}{*}{101} & \multirow[t]{2}{*}{$\mathrm{x}$} & $\begin{array}{l}\text { Psychosocial outcomes: inter- } \\
\text { vention improved fear and } \\
\text { worry about symptoms }(95 \% \\
\left.\text { CI } 0.39 \text { to } 1.09, d^{\mathrm{g}}=0.74\right) \text {, and } \\
\text { anxiety sensitivity }(95 \% \text { CI } \\
-0.07 \text { to } 0.47, d=0.20)\end{array}$ & $\begin{array}{l}\text { Psychosocial outcomes: } \\
\text { intervention improved fear } \\
\text { and worry about symptoms } \\
\text { (95\% CI } 0.59 \text { to } 1.59 \text {, } \\
d=1.05 \text { ) and anxiety sensi- } \\
\text { tivity ( } 95 \% \text { CI } 0.10 \text { to } \\
1.04, d=0.57 \text { ) at } 6 \text { months }\end{array}$ \\
\hline & & & & & $\begin{array}{l}\text { No significant results for per- } \\
\text { ceived stress or depressive } \\
\text { symptoms } \\
\text { Physical health outcomes: inter- } \\
\text { vention improved gastrointesti- } \\
\text { nal symptoms ( } 95 \% \text { CI } 0.16 \text { to } \\
0.84, d=0.50 \text { ) and pain intensity } \\
\text { and frequency ( } 95 \% \text { CI } 0.11 \text { to } \\
0.61, d=0.36 \text { ) }\end{array}$ & $\begin{array}{l}\text { No significant results for } \\
\text { perceived stress }(95 \% \text { CI } \\
-0.10 \text { to } 0.73, d=0.31 \text { ) or } \\
\text { depressive symptoms } \\
\text { (95\% CI }-0.14 \text { to } 0.46 \text {, } \\
d=0.16 \text { ) at } 6 \text { months } \\
\text { Physical health outcomes: } \\
\text { intervention improved } \\
\text { gastrointestinal symptoms } \\
(95 \% \text { CI } 0.24 \text { to } 1.02 \text {, } \\
d=0.63) \text { and pain intensity } \\
\text { and frequency }(95 \% \text { CI } \\
0.41 \text { to } 1.12, d=0.76) \text { at } 6 \\
\text { months }\end{array}$ \\
\hline \multirow[t]{3}{*}{$\begin{array}{l}\text { Move It Now } \\
{[47]}\end{array}$} & \multirow[t]{3}{*}{ Multisite RCT } & \multirow[t]{3}{*}{ Wait-list control } & \multirow[t]{3}{*}{69} & & $\begin{array}{l}\text { Psychosocial outcomes: inter- } \\
\text { vention improved rewarding } \\
\text { pain behavior by parents } \\
(P \leq .01) \text { and quality of life } \\
(P \leq .01 \text { to } .04, d=-0.87 \text { to } 0.34)\end{array}$ & $\begin{array}{l}\text { Psychosocial outcomes: } \\
\text { intervention improved } \\
\text { quality of life (besides } \\
\text { mental health subdomain) } \\
\text { at } 3 \text { months }\end{array}$ \\
\hline & & & & & $\begin{array}{l}\text { Physical health outcomes: inter- } \\
\text { vention improved pain intensity } \\
(P=.03, d=-0.42) \text {, pain interfer- }\end{array}$ & $\begin{array}{l}\text { No significant results for } \\
\text { rewarding pain behavior } \\
\text { by parents at } 3 \text { months }\end{array}$ \\
\hline & & & & & $\begin{array}{l}\text { ence }(P=.03, d=-0.46) \text { and } \\
\text { sleep problems }(P \leq .01 \\
d=-0.60)\end{array}$ & $\begin{array}{l}\text { Physical health outcomes: } \\
\text { no significant results for } \\
\text { pain intensity, pain interfer- } \\
\text { ence, or sleep problems at } \\
3 \text { months }\end{array}$ \\
\hline
\end{tabular}




\begin{tabular}{|c|c|c|c|c|c|c|}
\hline $\begin{array}{l}\text { Intervention } \\
\text { name }\end{array}$ & Type of study & Control group & $\begin{array}{l}\text { Sample } \\
\text { size }\end{array}$ & $\begin{array}{l}\text { Powered } \\
\text { for } \\
\text { analyses? }\end{array}$ & Posttreatment outcomes ${ }^{\text {a.b }}$ & Longitudinal outcomes ${ }^{\text {a.b }}$ \\
\hline TeenCope [59] & Multisite RCT & $\begin{array}{l}\text { eHealth managing } \\
\text { diabetes psychoedu- } \\
\text { cation for self-man- } \\
\text { agement }\end{array}$ & 320 & $\mathrm{x}$ & $\begin{array}{l}\text { Psychosocial outcomes: no sig- } \\
\text { nificant results for quality of } \\
\text { life, social acceptance, self-effi- } \\
\text { cacy, perceived stress, or dia- } \\
\text { betes family conflict } \\
\text { Physical health outcomes: no } \\
\text { significant results for } \mathrm{HbA}_{1 \mathrm{c}}{ }^{\mathrm{h}}\end{array}$ & $\begin{array}{l}\text { Psychosocial outcomes: no } \\
\text { significant results for } \\
\text { quality of life, social accep- } \\
\text { tance, self-efficacy, per- } \\
\text { ceived stress, or diabetes } \\
\text { family conflict at } 18 \\
\text { months } \\
\text { Physical health outcomes: } \\
\text { no significant results for } \\
\mathrm{HbA}_{1 \mathrm{c}} \text { at } 18 \text { months }\end{array}$ \\
\hline $\begin{array}{l}\text { Teens Taking } \\
\text { Charge: Manag- } \\
\text { ing Arthritis On- } \\
\text { line [52] }\end{array}$ & $\begin{array}{l}\text { Pilot multisite } \\
\text { RCT }\end{array}$ & Attentional control & 46 & & $\begin{array}{l}\text { Psychosocial outcomes: no sig- } \\
\text { nificant results for quality of } \\
\text { life, self-efficacy, or stress } \\
\text { Physical health outcomes: inter- } \\
\text { vention improved disease } \\
\text { knowledge }(P \leq .01, d=1.32) \text { and } \\
\text { pain intensity }(P=.03, d=0.78)\end{array}$ & N/A \\
\hline
\end{tabular}

Trautmann self- $\quad 3$-arm RCT Internet psychoedu- 65

help programs: cation intervention internet-based CBT, internetbased applied relaxation [54]
Internet psychoedu- 18 cation intervention

Psychosocial outcomes: no significant results for pain catastrophizing or psychological wellbeing

Physical health outcomes: no significant results for headache frequency and duration

Psychosocial outcomes: intervention improved pain catastrophizing $(P \leq .05)$

Physical health outcomes: intervention improved headache frequency $(P \leq .05)$

No significant results for headache intensity or headache duration

Psychosocial outcomes: intervention improved emotional functioning $(P=.04, d=-0.09)$ Physical health outcomes: no significant results for activity limitations, pain intensity, or sleep quality

\begin{tabular}{|c|c|c|c|c|}
\hline \multirow[t]{2}{*}{$\begin{array}{l}\text { Multisite RCT } \\
\text { [55] }\end{array}$} & \multirow[t]{2}{*}{$\begin{array}{l}\text { Internet education } \\
\text { control }\end{array}$} & \multirow{2}{*}{\multicolumn{2}{|c|}{273}} & $\begin{array}{l}\text { Psychosocial outcomes: inter- } \\
\text { vention improved emotional } \\
\text { functioning }(P=.04, d=-0.09)\end{array}$ \\
\hline & & & & $\begin{array}{l}\text { Physical health outcomes: no } \\
\text { significant results for activity } \\
\text { limitations, pain intensity, or } \\
\text { sleep quality }\end{array}$ \\
\hline
\end{tabular}

RCT [56] Wait-list control $48 \quad \mathrm{x}$

Psychosocial outcomes: no significant results for depression or parental protectiveness

Physical health outcomes: intervention improved activity limitations $\left(P \leq .01, \eta^{2 \mathrm{i}}=.17\right)$ and pain intensity $\left(P=.03, \eta^{2}=.11\right)$
Psychosocial outcomes: no significant results for pain catastrophizing or psychological well-being at 6 months

Physical health outcomes: no significant results for headache frequency and duration at 6 months

Psychosocial outcomes: intervention improved pain catastrophizing $(P \leq .05)$ at 6 months

Physical health outcomes: intervention improved headache frequency $(P \leq .05)$ at 6 months No significant results for headache intensity or headache duration at 6 months

Psychosocial outcomes: no significant results for emotional functioning at 6 months

Physical health outcomes: intervention improved activity limitations $(P=.03$; $d=-0.25)$ and sleep quality $(P=.04, d=0.16)$ at 6 months

No significant results for pain intensity at 6 months

Psychosocial outcomes: no significant results for depression and parental protectiveness at 3 months

Physical health outcomes: intervention improved activity limitations and pain intensity at 3 months 


\begin{tabular}{|c|c|c|c|c|c|c|}
\hline $\begin{array}{l}\text { Intervention } \\
\text { name }\end{array}$ & Type of study & Control group & $\begin{array}{l}\text { Sample } \\
\text { size }\end{array}$ & $\begin{array}{l}\text { Powered } \\
\text { for } \\
\text { analyses? }\end{array}$ & Posttreatment outcomes ${ }^{a \cdot b}$ & Longitudinal outcomes $^{\text {a.b }}$ \\
\hline & Pilot RCT [57] & $\begin{array}{l}\text { Specialized } \\
\text { headache treatment }\end{array}$ & 83 & $\mathrm{x}$ & $\begin{array}{l}\text { Psychosocial outcomes: no sig- } \\
\text { nificant results for anxiety or } \\
\text { depression } \\
\text { Physical health outcomes: no } \\
\text { significant results for headache } \\
\text { frequency, pain intensity, activ- } \\
\text { ity limitations, sleep duration, } \\
\text { or sleep efficiency }\end{array}$ & $\begin{array}{l}\text { Psychosocial outcomes: no } \\
\text { significant results for anxi- } \\
\text { ety or depression at } 3 \\
\text { months } \\
\text { Physical health outcomes: } \\
\text { no significant results for } \\
\text { headache frequency, pain } \\
\text { intensity, activity limita- } \\
\text { tions, sleep duration, or } \\
\text { sleep efficiency at } 3 \\
\text { months }\end{array}$ \\
\hline
\end{tabular}

${ }^{\mathrm{a}}$ Only analyses of between-group differences comparing the intervention and control arms are reported.

${ }^{\mathrm{b}}$ Information regarding confidence intervals, effect size, and $P$ values is included when reported in the original research publication.

${ }^{\mathrm{c}} \mathrm{RCT}$ : randomized controlled trial.

${ }^{\mathrm{d}} \mathrm{N} / \mathrm{A}$ : not applicable.

iCBT: internet-based cognitive behavioral therapy.

${ }^{\mathrm{f}}$ FGID: functional gastrointestinal disorder.

${ }^{\mathrm{g}} d$ : Cohen $d$.

${ }^{\mathrm{h}} \mathrm{HbA}_{1 \mathrm{c}}$ : hemoglobin $\mathrm{A}_{1 \mathrm{c}}$.

${ }^{\mathrm{i}} \eta^{2}$ : eta squared. 
Table 3. Original research publications with quantitative outcomes: nonrandomized controlled trials.

\begin{tabular}{|c|c|c|c|c|}
\hline $\begin{array}{l}\text { Intervention } \\
\text { name }\end{array}$ & $\begin{array}{l}\text { Type of } \\
\text { study }\end{array}$ & $\begin{array}{l}\text { Sample } \\
\text { size }\end{array}$ & Posttreatment outcomes ${ }^{\mathrm{a}}$ & Longitudinal outcomes ${ }^{\mathrm{a}}$ \\
\hline \multirow[t]{4}{*}{$\begin{array}{l}\text { ICBT }^{\mathrm{b}} \text { for adoles- } \\
\text { cents with FGID } \\
\text { [46] }\end{array}$} & \multirow[t]{4}{*}{ Pilot study } & \multirow[t]{4}{*}{29} & $\begin{array}{l}\text { Psychosocial outcomes: intervention improved stress } \\
\left(P<.05,95 \% \text { CI } 0.02 \text { to } 0.69, d^{\text {d }}=0.35\right) \text { at posttreat- } \\
\text { ment }\end{array}$ & $\begin{array}{l}\text { Psychosocial outcomes: intervention improved } \\
\text { anxiety }(P<.05,95 \% \text { CI } 0.08 \text { to } 0.81, d=0.44) \text { at } \\
6 \text { months }\end{array}$ \\
\hline & & & $\begin{array}{l}\text { No significant results for anxiety or depression at } \\
\text { posttreatment }\end{array}$ & $\begin{array}{l}\text { No significant results for depression or stress at } \\
6 \text { months }\end{array}$ \\
\hline & & & $\begin{array}{l}\text { Physical health outcomes: intervention improved } \\
\text { gastrointestinal symptoms }(P<.05,95 \% \text { CI } 2.37 \text { to } \\
10.58, d=0.50) \text {, pain interference }(P<.05,95 \% \text { CI } \\
0.11 \text { to } 0.61, d=0.36) \text {, and pain reactivity }(P<.05 \text {, } \\
95 \% \text { CI } 0.39 \text { to } 1.09, d=0.74) \text { at posttreatment }\end{array}$ & \multirow[t]{2}{*}{$\begin{array}{l}\text { Physical health outcomes: intervention improved } \\
\text { gastrointestinal symptoms ( } 95 \% \text { CI } 3.43 \text { to } 12.21 \text {, } \\
d=0.63) \text {, pain interference }(P<.05,95 \% \text { CI } 0.41 \\
\text { to } 1.12, d=0.76) \text {, pain reactivity }(P<.05,95 \% \text { CI } \\
0.59 \text { to } 1.59, d=1.05) \text {, and functional disability } \\
(P<.05,95 \% \text { CI } 0.10 \text { to } 1.04, d=0.56) \text { at } 6 \text { months }\end{array}$} \\
\hline & & & $\begin{array}{l}\text { No significant results for functional disability at } \\
\text { posttreatment }\end{array}$ & \\
\hline \multirow[t]{5}{*}{ PainSquad+ [49] } & \multirow[t]{5}{*}{$\begin{array}{l}\text { Multisite } \\
\text { pilot study }\end{array}$} & \multirow[t]{5}{*}{40} & $\begin{array}{l}\text { Psychosocial outcomes: intervention improved } \\
\text { emotional functioning }(P \leq .01, d=0.66) \text {, social func- }\end{array}$ & \multirow[t]{5}{*}{ N/A } \\
\hline & & & $\begin{array}{l}\text { tioning }(P \leq .01, d=0.46) \text {, and overall } \mathrm{HRQOL}^{\mathrm{e}} \\
(P=.02, d=0.43) \text { at posttreatment }\end{array}$ & \\
\hline & & & $\begin{array}{l}\text { No significant results for self-efficacy, or school } \\
\text { functioning at posttreatment }\end{array}$ & \\
\hline & & & $\begin{array}{l}\text { Physical health outcomes: intervention improved } \\
\text { pain intensity }(P \leq .01, d=0.67) \text { and pain interference } \\
(P=.03, d=0.38) \text { at posttreatment }\end{array}$ & \\
\hline & & & $\begin{array}{l}\text { No significant results for physical functioning at } \\
\text { posttreatment }\end{array}$ & \\
\hline \multirow[t]{2}{*}{ Onco-STEP [48] } & \multirow[t]{2}{*}{ Pilot study } & \multirow[t]{2}{*}{20} & \multirow{2}{*}{$\begin{array}{l}\text { Psychosocial outcomes: intervention improved post- } \\
\text { traumatic stress symptoms }(P \leq .01, d=0.63) \text {, anxiety } \\
(P \leq .01, d=0.74) \text {, fear of progression/relapse }(P<.05 \text {, } \\
d=0.48) \text {, and depression }(P \leq .01, d=1.0) \text { at posttreat- } \\
\text { ment }\end{array}$} & $\begin{array}{l}\text { Psychosocial outcomes: intervention improved } \\
\text { posttraumatic stress symptoms }(P<.01) \text {, fear of } \\
\text { progression/relapse }(P<.01) \text {, and anxiety }(P<.01) \\
\text { at } 3 \text { months }\end{array}$ \\
\hline & & & & No significant results for depression at 3 months \\
\hline \multirow[t]{3}{*}{$\begin{array}{l}\text { Web-based treat- } \\
\text { ment for adoles- } \\
\text { cents with IBD }{ }^{f} \\
{[58]}\end{array}$} & \multirow[t]{3}{*}{ Pilot study } & \multirow[t]{3}{*}{24} & \multirow{3}{*}{$\begin{array}{l}\text { Psychosocial outcomes: intervention improved somat- } \\
\text { ic symptoms }\left(P \leq .01, \eta^{2 \mathrm{~g}}=.41\right) \text {, approach coping } \\
\text { strategies }\left(P \leq .01, \eta^{2}=.43\right) \text {, distraction techniques } \\
\left(P \leq .01, \eta^{2}=.35\right) \text {, protective parenting behaviors } \\
\left(P=.03, \eta^{2}=.27\right) \text { at posttreatment } \\
\text { Physical health outcomes: no significant results for } \\
\text { abdominal pain at posttreatment }\end{array}$} & $\begin{array}{l}\text { Psychosocial outcomes: intervention improved } \\
\text { protective parenting behaviors }\left(P=.01, \eta^{2}=.44\right) \text { at } \\
6 \text { months }\end{array}$ \\
\hline & & & & $\begin{array}{l}\text { No significant results for somatic symptoms, ap- } \\
\text { proach coping strategies, or distraction techniques } \\
\text { at } 6 \text { months }\end{array}$ \\
\hline & & & & $\begin{array}{l}\text { Physical health outcomes: no significant results } \\
\text { for abdominal pain at } 6 \text { months }\end{array}$ \\
\hline
\end{tabular}

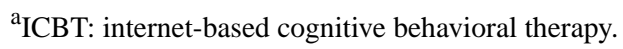

${ }^{\mathrm{b}}$ Information regarding confidence intervals, effect size, and $P$ values are included when reported in the original research publication.

${ }^{\mathrm{c}}$ FGID: functional gastrointestinal disorder.

$\mathrm{d} d$ : Cohen $d$.

${ }^{\mathrm{e}} \mathrm{HRQOL}$ : health-related quality of life.

IBD: inflammatory bowel disease.

${ }^{\mathrm{g}} \eta^{2}$ : eta squared.

A subset of $75 \%(12 / 16)$ of studies [45-48,53-59,61] evaluated longer term assessment time points ranging from 3 to 18 months (Tables 2 and 3). Nine studies (9/16, 56\%) $[45-48,53,55,56,58,61]$ showed promise of longer term gains from the intervention.

\section{Risk of Bias}

Risk of bias was evaluated for all included studies (Figure 2). Of the 16 studies, 12 reported random sequence generation and allocation concealment (ie, the pilot RCTs and RCTs). For the blinding of participants and personnel domain, 10 were high risk and 6 low risk; high-risk studies consisted of study designs with no control group or a wait-list control group. For the blinding of outcome assessment domain, 9 were high risk and 7 low risk. For attrition bias, 8 were low risk, 5 high risk, and 3 unclear; note that attrition rates for internet-based interventions (with an anchor point of around 50\%) tend to be higher than traditional face-to-face psychosocial interventions [65]. For selective reporting bias, studies were split in half between low risk and unclear; studies were rated as unclear due to a lack of clinical trial registration or published protocol. For other biases, 12 were considered low risk and 4 high risk. 
Figure 2. Risk of bias.

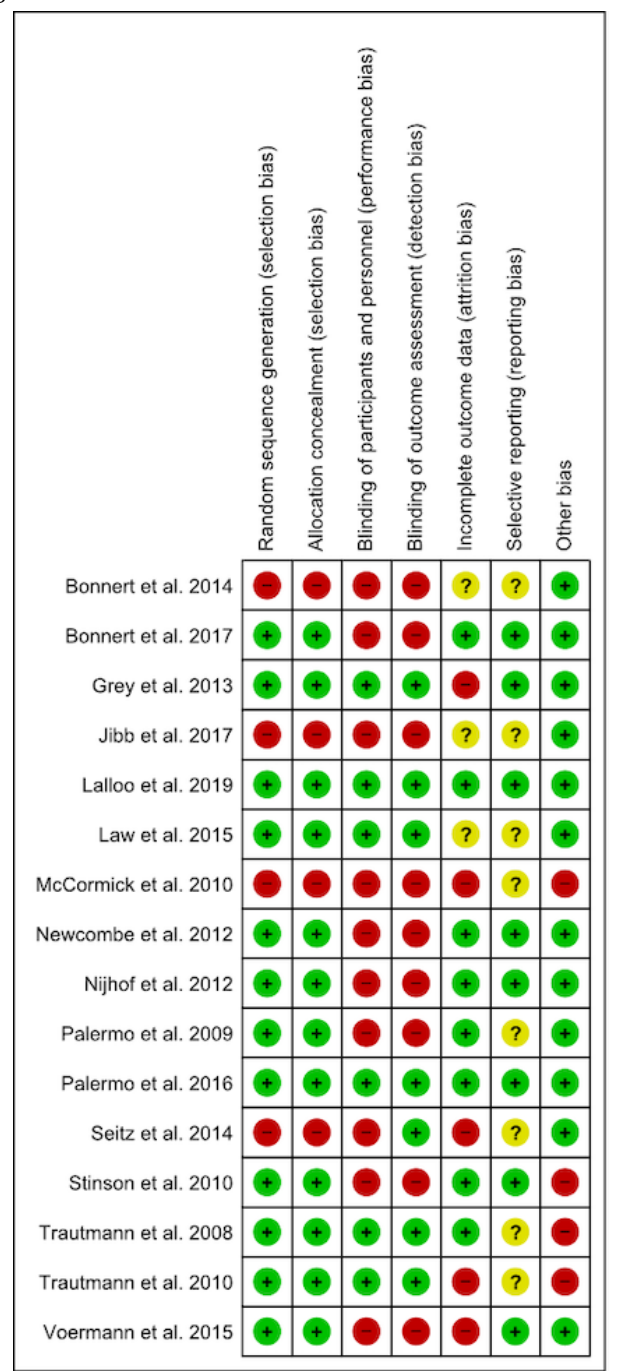

\section{Discussion}

\section{Overview}

A majority of youth with chronic illnesses struggle with issues that psychosocial interventions effectively manage such as anxiety, stress, depression, maladjustment, pain, and poor coping skills $[4,7]$. Within the past decade, digital health interventions have been increasingly popular with widespread access to the internet and smartphones. To our knowledge, this is the first systematic review summarizing the research evidence in support of the efficacy of eHealth and mHealth psychosocial interventions for youth chronic illnesses. Each of the interventions identified was designed for a specific chronic illness population. The state of the science is still in its nascent stages, with only 16 published efficacy studies of 12 unique interventions identified. We provided information on the structure and content of included interventions and relationships between each of the interventions and various psychosocial and health outcomes. Given the substantial number of studies in which full articles were reviewed for eligibility ( $n=133$, Figure 1 ), this points to growing interest in digital interventions for youth with chronic illnesses. Few digital interventions have advanced to the stages of efficacy testing $(n=16)$, and even fewer in an RCT with an active comparison condition $(n=8)$. This systematic review suggests that disseminating traditional evidence-based psychotherapies via novel digital health technologies may be efficacious.

Consistent with evidence-based digital health practices and recent systematic reviews of digital interventions with youth chronic illness and mental health populations [17,24,25,28,33], our systematic review of eHealth and mHealth psychosocial interventions for youth chronic illness populations found (a) early evidence of improvements in psychosocial and physical health outcomes immediately posttreatment; (b) early evidence of the maintenance of treatment gains at longer term follow-up extending to $3+$ months; (c) a prevalence of computerized cognitive behavioral therapy interventions; (d) varying levels of psychosocial staff support via text, email, phone, online discussion forums, or private online messaging centers; and (e) key methodological limitations for a majority of included studies such as lack of blinded outcomes assessment, limited number of RCTs, and few studies with active treatment comparison groups.

This review also suggests several gaps in the existing literature. Future research should focus on optimizing digital intervention design and implementation, namely how to efficiently streamline resource-intensive personnel assistance and encourage self-direction in order to sustain intervention efficacy and 
engagement while minimizing costs [66]. Notably, only two of the included interventions did not involve some form of contact with providers or research staff. In addition to clinician involvement, given that half of the interventions reviewed included caregiver support, new digital intervention research should continue to explore the additive value of caregiver involvement where appropriate with unique content designed for parent caregivers $[55,56]$. Next, as each of the interventions included in our review was designed for a specific chronic illness, further research is needed to ascertain whether a disease nonspecific transdiagnostic approach $[67,68]$ to designing digital psychosocial interventions applicable across heterogeneous disease groups is warranted. In particular, online mindfulness-based interventions have been shown to be successful in chronic illness and other populations [69-71]. Interventions also had multiple components, which may benefit from dismantling studies to identify which active therapeutic ingredients lead to positive outcomes [72]. Similarly, emphasis should be placed on identifying and targeting the aspects of digital engagement that lead to the intended behavior change rather than just encouraging more frequent use [73]. The use of analytics in eHealth and mHealth can provide invaluable insights into active therapeutic ingredients, aspects of effective digital engagement, treatment moderators (for whom interventions work), and treatment mediators (how treatments work) [74,75]. Finally, future studies might assess the value of more novel technologically enabled features such as just-in-time adaptive intervention designs (JITAIs). Indeed, JITAIs use causal modeling to identify the appropriate type and dose of intervention at the optimal point in time by measuring and responding to an individual's changing health states [76,77]. These innovative programs have the potential to meet evolving real-time needs of youth at risk.

Given the interest in and rapidly changing landscape of digital health, it is likely that new publications have been released during the typical time frame it had taken us to rigorously complete our systematic review. We intend to publish a 5-year systematic review update populated with new efficacy studies and trials. The median timing of Cochrane systematic review updates is greater than 5 years [78]. Although previous research suggests only $4 \%$ of systematic review updates report a change in conclusions [79], our overarching findings may be subject to change given the limited number of included studies and the quickly evolving digital health landscape.

\section{Limitations}

Limitations to consider are as follows. First, most of the interventions were designed and tested as web-based eHealth interventions, with only two mHealth intervention designed for use on smartphone devices. We anticipate that more mHealth interventions will be tested for efficacy and come down the pipeline in the years to come as smartphones become more and more ubiquitous. Previous research suggests that mHealth apps are widely accepted by the general public for coping skills and stress management, and beliefs and attitudes toward mHealth are positive [80]. Second, the majority of studies were not powered to detect meaningful changes in health outcomes of interest nor did they designate primary versus secondary outcomes in their research designs. Third, only half of the studies used an active treatment comparison condition or blinded participants to assigned treatment condition, and findings of favorable treatment response may be susceptible to the placebo effect [81]. Fourth, there exists the possibility of publication bias because nonsignificant findings are often difficult to publish. Fifth, although our search was executed by a medical librarian in five well-established library databases, other databases to which our university does not have access (eg, Scopus) may have uncovered additional relevant publications. Finally, given the heterogeneity of disease groups, measurement time points, and study outcomes measures, it was not possible to conduct a meta-analysis and provide synthesized results of the efficacy studies included in our systematic review.

\section{Conclusions}

The strengths of this paper include the systematic approach to synthesizing the great breadth of literature across pediatric illness populations and the eHealth and mHealth focus increasingly popular among youths. This publication is especially timely given heightened psychological distress and exacerbating mental health symptoms for youth in the context of the COVID-19 pandemic, in-home confinement, school closures, social distancing, and a shift toward online and telehealth services [82-85]. We found intervention features unique to digital platforms such as gamification, ecological momentary assessment, and machine learning algorithms. Such features capitalize on technological advances to intervene during distressing situations in real time and tailor content to individual preferences and needs. Leveraging such technological advances allows movement toward a data-driven and personalized approach to precision mental health [86]. The state of the science is still in its early stages, and further clinical trials research is needed to confirm whether evidence-based psychosocial interventions traditionally delivered in-person may be successfully translated to digital formats for a range of youth chronic illness populations.

\section{Acknowledgments}

NL is funded as an Implementation Science Scholar through the National Heart, Lung, and Blood Institute of the National Institutes of Health (grant number: 5K12 HL137940-02).

\section{Conflicts of Interest}

None declared. 


\section{Multimedia Appendix 1}

Primary search strategy.

[DOCX File, 22 KB-Multimedia Appendix 1]

\section{Multimedia Appendix 2}

Supplemental search strategy.

[DOCX File, 20 KB-Multimedia Appendix 2]

\section{Multimedia Appendix 3}

Intervention and personnel-supported components.

[DOCX File, $21 \mathrm{~KB}-$ Multimedia Appendix 3]

\section{References}

1. Van Cleave J, Gortmaker SL, Perrin JM. Dynamics of obesity and chronic health conditions among children and youth. JAMA 2010 Feb 17;303(7):623-630. [doi: 10.1001/jama.2010.104] [Medline: 20159870]

2. van Dyck PC, Kogan MD, McPherson MG, Weissman GR, Newacheck PW. Prevalence and characteristics of children with special health care needs. Arch Pediatr Adolesc Med 2004 Sep;158(9):884-890. [doi: 10.1001/archpedi.158.9.884] [Medline: 15351754 ]

3. Miller GF, Coffield E, Leroy Z, Wallin R. Prevalence and costs of five chronic conditions in children. J Sch Nurs 2016 Oct;32(5):357-364 [FREE Full text] [doi: 10.1177/1059840516641190] [Medline: 27044668]

4. Wallander JL, Varni JW. Effects of pediatric chronic physical disorders on child and family adjustment. J Child Psychol Psychiatry 1998 Jan;39(1):29-46. [Medline: 9534085]

5. Yeo M, Sawyer S. Chronic illness and disability. BMJ 2005 Mar 26;330(7493):721-723 [FREE Full text] [doi: 10.1136/bmj.330.7493.721] [Medline: 15790645$]$

6. Warschburger P, Hänig J, Friedt M, Posovszky C, Schier M, Calvano C. Health-related quality of life in children with abdominal pain due to functional or organic gastrointestinal disorders. J Pediatr Psychol 2014;39(1):45-54. [doi: 10.1093/jpepsy/jst070] [Medline: 24055816]

7. Butler A, Van Lieshout RJ, Lipman EL, MacMillan HL, Gonzalez A, Gorter JW, et al. Mental disorder in children with physical conditions: a pilot study. BMJ Open 2018 Dec 03;8(1):e019011. [doi: 10.1136/bmjopen-2017-019011] [Medline: 29301763]

8. Kieling C, Baker-Henningham H, Belfer M, Conti G, Ertem I, Omigbodun O, et al. Child and adolescent mental health worldwide: evidence for action. Lancet 2011 Oct 22;378(9801):1515-1525. [doi: 10.1016/S0140-6736(11)60827-1] [Medline: 22008427]

9. Compas BE, Jaser SS, Dunn MJ, Rodriguez EM. Coping with chronic illness in childhood and adolescence. Annu Rev Clin Psychol 2012;8:455-480 [FREE Full text] [doi: 10.1146/annurev-clinpsy-032511-143108] [Medline: 22224836]

10. Ruiz F. Acceptance and commitment therapy versus traditional cognitive behavioral therapy: a systematic review and meta-analysis of current empirical evidence. In J Psychol Psychologic Ther 2012;12(3):333-358 [FREE Full text]

11. Tark R, Metelitsa M, Akkermann K, Saks K, Mikkel S, Haljas K. Usability, acceptability, feasibility, and effectiveness of a gamified mobile health intervention (Triumf) for pediatric patients: qualitative study. JMIR Serious Games 2019 Sep 30;7(3):e13776 [FREE Full text] [doi: 10.2196/13776] [Medline: 31573904]

12. Pai ALH, McGrady M. Systematic review and meta-analysis of psychological interventions to promote treatment adherence in children, adolescents, and young adults with chronic illness. J Pediatr Psychol 2014 Sep;39(8):918-931. [doi: 10.1093/jpepsy/jsu038] [Medline: 24952359]

13. Barlow JH, Ellard DR. Psycho-educational interventions for children with chronic disease, parents and siblings: an overview of the research evidence base. Child Care Health Dev 2004 Nov;30(6):637-645. [doi: 10.1111/j.1365-2214.2004.00474.x] [Medline: 15527474$]$

14. Mojtabai R, Olfson M. National trends in mental health care for US adolescents. JAMA Psychiatry 2020 Jul 01;77(7):703-714. [doi: 10.1001/jamapsychiatry.2020.0279] [Medline: 32211824]

15. Cama S, Malowney M, Smith AJB, Spottswood M, Cheng E, Ostrowsky L, et al. Availability of outpatient mental health care by pediatricians and child psychiatrists in five U.S. cities. Int J Health Serv 2017 Oct;47(4):621-635. [doi: 10.1177/0020731417707492] [Medline: 28474997]

16. Ybarra ML, Eaton WW. Internet-based mental health interventions. Ment Health Serv Res 2005 Jun;7(2):75-87. [Medline: 15974154]

17. Ebert DD, Zarski A, Christensen H, Stikkelbroek Y, Cuijpers P, Berking M, et al. Internet and computer-based cognitive behavioral therapy for anxiety and depression in youth: a meta-analysis of randomized controlled outcome trials. PLoS One 2015;10(3):e0119895 [FREE Full text] [doi: 10.1371/journal.pone.0119895] [Medline: 25786025] 
18. Granger D, Vandelanotte C, Duncan MJ, Alley S, Schoeppe S, Short C, et al. Is preference for mHealth intervention delivery platform associated with delivery platform familiarity? BMC Public Health 2016 Jul 22;16:619 [FREE Full text] [doi: 10.1186/s12889-016-3316-2] [Medline: 27450240]

19. Gulliver A, Griffiths KM, Christensen H. Perceived barriers and facilitators to mental health help-seeking in young people: a systematic review. BMC Psychiatry 2010;10:113 [FREE Full text] [doi: 10.1186/1471-244X-10-113] [Medline: 21192795]

20. Gray NJ, Klein JD, Noyce PR, Sesselberg TS, Cantrill JA. Health information-seeking behaviour in adolescence: the place of the internet. Soc Sci Med 2005 Apr;60(7):1467-1478. [doi: 10.1016/j.socscimed.2004.08.010] [Medline: 15652680]

21. Spek V, Cuijpers P, Nyklícek I, Riper H, Keyzer J, Pop V. Internet-based cognitive behaviour therapy for symptoms of depression and anxiety: a meta-analysis. Psychol Med 2007 Mar;37(3):319-328. [doi: 10.1017/S0033291706008944] [Medline: 17112400 ]

22. Clarke AM, Kuosmanen T, Barry MM. A systematic review of online youth mental health promotion and prevention interventions. J Youth Adolesc 2015 Jan;44(1):90-113. [doi: 10.1007/s10964-014-0165-0] [Medline: 25115460]

23. Proudfoot J, Parker G, Hadzi PD, Manicavasagar V, Adler E, Whitton A. Community attitudes to the appropriation of mobile phones for monitoring and managing depression, anxiety, and stress. J Med Internet Res 2010;12(5):e64 [FREE Full text] [doi: 10.2196/jmir.1475] [Medline: 21169174]

24. Fisher E, Law E, Dudeney J, Eccleston C, Palermo TM. Psychological therapies (remotely delivered) for the management of chronic and recurrent pain in children and adolescents. Cochrane Database Syst Rev 2019 Apr 02;4:CD011118 [FREE Full text] [doi: 10.1002/14651858.CD011118.pub3] [Medline: 30939227]

25. Ramsey RR, Plevinsky JM, Kollin SR, Gibler RC, Guilbert TW, Hommel KA. Systematic review of digital interventions for pediatric asthma management. J Allergy Clin Immunol Pract 2020 Apr;8(4):1284-1293. [doi: 10.1016/j.jaip.2019.12.013] [Medline: $\underline{31870809]}$

26. Nickels A, Dimov V. Innovations in technology: social media and mobile technology in the care of adolescents with asthma. Curr Allergy Asthma Rep 2012 Dec;12(6):607-612. [doi: 10.1007/s11882-012-0299-7] [Medline: 22976493]

27. Alquran A, Lambert KA, Farouque A, Holland A, Davies J, Lampugnani ER, et al. Smartphone applications for encouraging asthma self-management in adolescents: a systematic review. Int J Environ Res Public Health 2018 Dec 29;15(11) [FREE Full text] [doi: 10.3390/ijerph15112403] [Medline: 30380692]

28. Hollis C, Falconer CJ, Martin JL, Whittington C, Stockton S, Glazebrook C, et al. Annual research review: digital health interventions for children and young people with mental health problems: a systematic and meta-review. J Child Psychol Psychiatry 2016 Dec 10;58(4):474-503. [doi: 10.1111/jcpp.12663] [Medline: 27943285]

29. Grist R, Porter J, Stallard P. Mental health mobile apps for preadolescents and adolescents: a systematic review. J Med Internet Res 2017 May 25;19(5):e176 [FREE Full text] [doi: 10.2196/jmir.7332] [Medline: 28546138]

30. Vigerland S, Lenhard F, Bonnert M, Lalouni M, Hedman E, Ahlen J, et al. Internet-delivered cognitive behavior therapy for children and adolescents: a systematic review and meta-analysis. Clin Psychol Rev 2016 Dec;50:1-10 [FREE Full text] [doi: 10.1016/j.cpr.2016.09.005] [Medline: 27668988]

31. Thabrew H, Stasiak K, Hetrick SE, Wong S, Huss JH, Merry SN. E-Health interventions for anxiety and depression in children and adolescents with long-term physical conditions. Cochrane Database Syst Rev 2018 Aug 15;8:CD012489 [FREE Full text] [doi: 10.1002/14651858.CD012489.pub2] [Medline: 30110718]

32. Low JK, Manias E. Use of technology-based tools to support adolescents and young adults with chronic disease: systematic review and meta-analysis. JMIR Mhealth Uhealth 2019 Jul 18;7(7):e12042 [FREE Full text] [doi: 10.2196/12042] [Medline: 31322129]

33. Canter KS, Christofferson J, Scialla MA, Kazak AE. Technology-focused family interventions in pediatric chronic illness: a systematic review. J Clin Psychol Med Settings 2018 Apr 20;26(1):68-87. [doi: 10.1007/s10880-018-9565-8] [Medline: 29679264]

34. Rousseau GK, Jamieson BA, Rogers WA, Mead SE, Sit RA. Assessing the usability of on-line library systems. Behav Inf Technol 1998 Jan;17(5):274-281. [doi: 10.1080/014492998119346]

35. Bernell S, Howard SW. Use your words carefully: what is a chronic disease? Front Public Health 2016;4:159 [FREE Full text] [doi: 10.3389/fpubh.2016.00159] [Medline: 27532034]

36. Law E, Fisher E, Eccleston C, Palermo TM. Psychological interventions for parents of children and adolescents with chronic illness. Cochrane Database Syst Rev 2019 Mar 18;3:CD009660 [FREE Full text] [doi: 10.1002/14651858.CD009660.pub4] [Medline: $\underline{30883665]}$

37. Scaturo DJ. The evolution of psychotherapy and the concept of manualization: an integrative perspective. Prof Psychol Res Pract 2001;32(5):522-530. [doi: 10.1037/0735-7028.32.5.522]

38. McFarlane AC. The long-term costs of traumatic stress: intertwined physical and psychological consequences. World Psychiatry 2010 Feb;9(1):3-10. [doi: 10.1002/j.2051-5545.2010.tb00254.x] [Medline: 20148146]

39. Vannatta K, Gerhardt C. Pediatric oncology: psychosocial outcomes for children and families. In: Roberts MC, editor. Handbook of Pediatric Psychology. New York: Guilford Press; 2003:342-357.

40. Hanson CL, Henggeler SW, Burghen GA. Model of associations between psychosocial variables and health-outcome measures of adolescents with IDDM. Diabetes Care 1987;10(6):752-758. [doi: 10.2337/diacare.10.6.752] [Medline: 3428051] 
41. Livneh H. Psychosocial adaptation to chronic illness and disability. Rehabil Couns Bull 2016 Aug 13;44(3):151-160. [doi: $10.1177 / 003435520104400305]$

42. Higgins JPT, Altman DG, Gøtzsche PC, Jüni P, Moher D, Oxman AD, et al. The Cochrane Collaboration's tool for assessing risk of bias in randomised trials. BMJ 2011;343:d5928 [FREE Full text] [Medline: 22008217]

43. Higgins J, Thomas J, Chandler J. Cochrane Handbook for Systematic Reviews of Interventions. Hoboken: John Wiley \& Sons; 2019.

44. Newcombe PA, Dunn TL, Casey LM, Sheffield JK, Petsky H, Anderson-James S, et al. Breathe Easier Online: evaluation of a randomized controlled pilot trial of an Internet-based intervention to improve well-being in children and adolescents with a chronic respiratory condition. J Med Internet Res 2012 Feb 08;14(1):e23 [FREE Full text] [doi: 10.2196/jmir.1997] [Medline: 22356732]

45. Nijhof SL, Bleijenberg G, Uiterwaal CSPM, Kimpen JLL, van de Putte EM. Effectiveness of internet-based cognitive behavioural treatment for adolescents with chronic fatigue syndrome (FITNET): a randomised controlled trial. Lancet 2012 Apr 14;379(9824):1412-1418. [doi: 10.1016/S0140-6736(12)60025-7] [Medline: 22385683]

46. Bonnert M, Ljótsson B, Hedman E, Andersson J, Arnell H, Benninga MA, et al. Internet-delivered cognitive behavior therapy for adolescents with functional gastrointestinal disorders: an open trial. Internet Interv 2014 Jul;1(3):141-148. [doi: 10.1016/j.invent.2014.07.002]

47. Voerman JS, Remerie S, Westendorp T, Timman R, Busschbach JJV, Passchier J, et al. Effects of a guided internet-delivered self-help intervention for adolescents with chronic pain. J Pain 2015 Nov;16(11):1115-1126. [doi: 10.1016/j.jpain.2015.07.011] [Medline: 26281947]

48. Seitz DCM, Knaevelsrud C, Duran G, Waadt S, Loos S, Goldbeck L. Efficacy of an internet-based cognitive-behavioral intervention for long-term survivors of pediatric cancer: a pilot study. Support Care Cancer 2014 Aug;22(8):2075-2083. [doi: 10.1007/s00520-014-2193-4] [Medline: 24639036]

49. Jibb LA, Stevens BJ, Nathan PC, Seto E, Cafazzo JA, Johnston DL, et al. Implementation and preliminary effectiveness of a real-time pain management smartphone app for adolescents with cancer: a multicenter pilot clinical study. Pediatr Blood Cancer 2017 Oct;64(10). [doi: 10.1002/pbc.26554] [Medline: 28423223]

50. Jibb LA, Stevens BJ, Nathan PC, Seto E, Cafazzo JA, Johnston DL, et al. Perceptions of adolescents with cancer related to a pain management app and its evaluation: qualitative study nested within a multicenter pilot feasibility study. JMIR Mhealth Uhealth 2018 Apr 06;6(4):e80 [FREE Full text] [doi: 10.2196/mhealth.9319] [Medline: 29625951]

51. Whittemore R, Jaser SS, Faulkner MS, Murphy K, Delamater A, Grey M. Type 1 diabetes eHealth psychoeducation: youth recruitment, participation, and satisfaction. J Med Internet Res 2013;15(1):e15 [FREE Full text] [doi: 10.2196/jmir.2170] [Medline: 23360729]

52. Stinson J, McGrath P, Hodnett E, Feldman B, Duffy C, Huber A, et al. Usability testing of an online self-management program for adolescents with juvenile idiopathic arthritis. J Med Internet Res 2010;12(3):e30 [FREE Full text] [doi: 10.2196/jmir.1349] [Medline: 20675293]

53. Trautmann E, Kröner-Herwig B. Internet-based self-help training for children and adolescents with recurrent headache: a pilot study. Behav Cogn Psychother 2008 Apr 15;36(2):241-245. [doi: 10.1017/s1352465808004219]

54. Trautmann E, Kröner-Herwig B. A randomized controlled trial of Internet-based self-help training for recurrent headache in childhood and adolescence. Behav Res Ther 2010 Jan;48(1):28-37. [doi: 10.1016/j.brat.2009.09.004] [Medline: 19782343]

55. Palermo TM, Law EF, Fales J, Bromberg MH, Jessen-Fiddick T, Tai G. Internet-delivered cognitive-behavioral treatment for adolescents with chronic pain and their parents: a randomized controlled multicenter trial. Pain 2016 Jan;157(1):174-185 [FREE Full text] [doi: 10.1097/j.pain.0000000000000348] [Medline: 26335910]

56. Palermo TM, Wilson AC, Peters M, Lewandowski A, Somhegyi H. Randomized controlled trial of an Internet-delivered family cognitive-behavioral therapy intervention for children and adolescents with chronic pain. Pain 2009 Nov;146(1-2):205-213 [FREE Full text] [doi: 10.1016/j.pain.2009.07.034] [Medline: 19695776]

57. Law EF, Beals-Erickson SE, Noel M, Claar R, Palermo TM. Pilot randomized controlled trial of internet-delivered cognitive-behavioral treatment for pediatric headache. Headache 2015;55(10):1410-1425 [FREE Full text] [doi: 10.1111/head.12635] [Medline: 26316194]

58. McCormick M, Reed-Knight B, Lewis JD, Gold BD, Blount RL. Coping skills for reducing pain and somatic symptoms in adolescents with IBD. Inflamm Bowel Dis 2010 Dec;16(12):2148-2157. [doi: 10.1002/ibd.21302] [Medline: 20848505]

59. Grey M, Whittemore R, Jeon S, Murphy K, Faulkner MS, Delamater A, et al. Internet psycho-education programs improve outcomes in youth with type 1 diabetes. Diabetes Care 2013 Sep;36(9):2475-2482 [FREE Full text] [doi: 10.2337/dc12-2199] [Medline: 23579179]

60. Lalloo C, Hundert A, Harris L, Pham Q, Campbell F, Chorney J, et al. Capturing daily disease experiences of adolescents with chronic pain: mhealth-mediated symptom tracking. JMIR Mhealth Uhealth 2019 Jan 17;7(1):e11838 [ㅍEE Full text] [doi: 10.2196/11838] [Medline: 30664472]

61. Bonnert M, Olén O, Lalouni M, Benninga MA, Bottai M, Engelbrektsson J, et al. Internet-delivered cognitive behavior therapy for adolescents with irritable bowel syndrome: a randomized controlled trial. Am J Gastroenterol 2017 Jan;112(1):152-162. [doi: 10.1038/ajg.2016.503] [Medline: 27845338] 
62. Hofmann SG, Asnaani A, Vonk IJJ, Sawyer AT, Fang A. The efficacy of cognitive behavioral therapy: a review of meta-analyses. Cognit Ther Res 2012 Oct 1;36(5):427-440 [FREE Full text] [doi: 10.1007/s10608-012-9476-1] [Medline: 23459093]

63. Sperry L. Treatment of Chronic Medical Conditions: Cognitive-Behavioral Therapy Strategies and Integrative Treatment Protocols. Washington: American Psychological Association; 2009.

64. Firth J, Torous J, Yung AR. Ecological momentary assessment and beyond: the rising interest in e-mental health research. J Psychiatr Res 2016 Sep;80:3-4 [FREE Full text] [doi: 10.1016/j.jpsychires.2016.05.002] [Medline: 27236099]

65. Eysenbach G. The law of attrition. J Med Internet Res 2005;7(1):e11 [FREE Full text] [doi: 10.2196/jmir.7.1.e11] [Medline: 15829473]

66. Mohr DC, Burns MN, Schueller SM, Clarke G, Klinkman M. Behavioral intervention technologies: evidence review and recommendations for future research in mental health. Gen Hosp Psychiatry 2013 Aug;35(4):332-338 [REEE Full text] [doi: 10.1016/j.genhosppsych.2013.03.008] [Medline: 23664503]

67. Barlow DH, Farchione TJ, Bullis JR, Gallagher MW, Murray-Latin H, Sauer-Zavala S, et al. The unified protocol for transdiagnostic treatment of emotional disorders compared with diagnosis-specific protocols for anxiety disorders: a randomized clinical trial. JAMA Psychiatry 2017 Aug 02;74(9):875-884. [doi: 10.1001/jamapsychiatry.2017.2164] [Medline: 28768327]

68. Steele SJ, Farchione TJ, Cassiello-Robbins C, Ametaj A, Sbi S, Sauer-Zavala S, et al. Efficacy of the unified protocol for transdiagnostic treatment of comorbid psychopathology accompanying emotional disorders compared to treatments targeting single disorders. J Psychiatr Res 2018 Sep;104:211-216 [FREE Full text] [doi: 10.1016/j.jpsychires.2018.08.005] [Medline: 30103069]

69. Abujaradeh H, Safadi R, Sereika SM, Kahle CT, Cohen SM. Mindfulness-based interventions among adolescents with chronic diseases in clinical settings: a systematic review. J Pediatr Health Care 2018;32(5):455-472. [doi: 10.1016/j.pedhc.2018.04.001] [Medline: 29941236]

70. Zou L, Yeung A, Quan X, Boyden SD, Wang H. A systematic review and meta-analysis of mindfulness-based (Baduanjin) exercise for alleviating musculoskeletal pain and improving sleep quality in people with chronic diseases. Int J Environ Res Public Health 2018 Jan 25;15(2) [FREE Full text] [doi: 10.3390/ijerph15020206] [Medline: 29370149]

71. Ahmad F, Wang J, El Morr C. Online mindfulness interventions: a systematic review. In: Ahmad F, Wang J, El Morr C, editors. Novel Applications of Virtual Communities in Healthcare Settings. Hershey: IGI Global; 2018:1-27.

72. Papa A, Follette W. Dismantling studies of psychotherapy. In: Papa A, Follette W, editors. The Encyclopedia of Clinical Psychology. Hoboken: John Wiley \& Sons; 2014:1-6.

73. Yardley L, Spring BJ, Riper H, Morrison LG, Crane DH, Curtis K, et al. Understanding and promoting effective engagement with digital behavior change interventions. Am J Prev Med 2016 Nov;51(5):833-842. [doi: 10.1016/j.amepre.2016.06.015] [Medline: 27745683]

74. El Morr C, Ali-Hassan H. Analytics in Healthcare: A Practical Introduction. Berlin: Springer; 2019.

75. El Morr C, Ritvo P, Ahmad F, Moineddin R. Effectiveness of an 8-week web-based mindfulness virtual community intervention for university students on symptoms of stress, anxiety, and depression: randomized controlled trial. JMIR Ment Health 2020 Jul 17;7(7):e18595 [FREE Full text] [doi: 10.2196/18595] [Medline: 32554380]

76. Nahum-Shani I, Smith SN, Spring BJ, Collins LM, Witkiewitz K, Tewari A, et al. Just-in-time adaptive interventions (JITAIs) in mobile health: key components and design principles for ongoing health behavior support. Ann Behav Med 2018 May 18;52(6):446-462 [FREE Full text] [doi: 10.1007/s12160-016-9830-8] [Medline: 27663578]

77. Klasnja P, Hekler EB, Shiffman S, Boruvka A, Almirall D, Tewari A, et al. Microrandomized trials: an experimental design for developing just-in-time adaptive interventions. Health Psychol 2015 Dec;34 Suppl:1220-1228. [doi: 10.1037/hea0000305] [Medline: 26651463]

78. Bashir R, Surian D, Dunn AG. Time-to-update of systematic reviews relative to the availability of new evidence. Syst Rev 2018 Nov 17;7(1):195 [FREE Full text] [doi: 10.1186/s13643-018-0856-9] [Medline: 30447694]

79. French SD, McDonald S, McKenzie JE, Green SE. Investing in updating: how do conclusions change when Cochrane systematic reviews are updated? BMC Med Res Methodol 2005 Oct 14;5:33 [FREE Full text] [doi: 10.1186/1471-2288-5-33] [Medline: 16225692$]$

80. Apolinário-Hagen J, Hennemann S, Fritsche L, Drüge M, Breil B. Determinant factors of public acceptance of stress management apps: survey study. JMIR Ment Health 2019 Nov 07;6(11):e15373 [FREE Full text] [doi: 10.2196/15373] [Medline: $\underline{\text { 31697243] }}$

81. Price DD, Finniss DG, Benedetti F. A comprehensive review of the placebo effect: recent advances and current thought. Annu Rev Psychol 2008;59:565-590. [doi: 10.1146/annurev.psych.59.113006.095941] [Medline: 17550344]

82. Ho CS, Chee CY, Ho RC. Mental health strategies to combat the psychological impact of COVID-19 beyond paranoia and panic. Ann Acad Med Singapore 2020 Jan;49(1):1-3 [FREE Full text] [Medline: 32200399]

83. Liu S, Yang L, Zhang C, Xiang Y, Liu Z, Hu S, et al. Online mental health services in China during the COVID-19 outbreak. Lancet Psychiatry 2020 Apr;7(4):e17-e18. [doi: 10.1016/S2215-0366(20)30077-8] [Medline: 32085841] 
84. Wang G, Zhang Y, Zhao J, Zhang J, Jiang F. Mitigate the effects of home confinement on children during the COVID-19 outbreak. Lancet 2020 Mar 21;395(10228):945-947 [FREE Full text] [doi: 10.1016/S0140-6736(20)30547-X] [Medline: 32145186]

85. Golberstein E, Wen H, Miller BF. Coronavirus disease 2019 (COVID-19) and mental health for children and adolescents. JAMA Pediatr 2020 Apr 14. [doi: 10.1001/jamapediatrics.2020.1456] [Medline: $\underline{32286618}$ ]

86. Bickman L, Lyon AR, Wolpert M. Achieving precision mental health through effective assessment, monitoring, and feedback processes: introduction to the special issue. Adm Policy Ment Health 2016 May;43(3):271-276 [FREE Full text] [doi: 10.1007/s10488-016-0718-5] [Medline: 26887937]
Abbreviations
JITAI: just-in-time adaptive intervention design
mHealth: mobile health apps
PRISMA: preferred reporting items for systematic reviews and meta-analyses
RCT: randomized controlled trial

\author{
Edited by S Badawy; submitted 08.07.20; peer-reviewed by A Chen, C El Morr, S Ouhbi; comments to author 11.08.20; revised version \\ received 02.10.20; accepted 16.10.20; published 10.11.20 \\ Please cite as: \\ Lau N, Waldbaum S, Parigoris R, O'Daffer A, Walsh C, Colt SF, Yi-Frazier JP, Palermo TM, McCauley E, Rosenberg AR \\ eHealth and mHealth Psychosocial Interventions for Youths With Chronic Illnesses: Systematic Review \\ JMIR Pediatr Parent 2020;3(2):e22329 \\ URL: http://pediatrics.jmir.org/2020/2/e22329/ \\ doi: 10.2196/22329 \\ PMID: $\underline{3075743}$
}

CNancy Lau, Shayna Waldbaum, Ryan Parigoris, Alison O'Daffer, Casey Walsh, Susannah F Colt, Joyce P Yi-Frazier, Tonya M Palermo, Elizabeth McCauley, Abby R Rosenberg. Originally published in JMIR Pediatrics and Parenting (http://pediatrics.jmir.org), 10.11.2020. This is an open-access article distributed under the terms of the Creative Commons Attribution License (https://creativecommons.org/licenses/by/4.0/), which permits unrestricted use, distribution, and reproduction in any medium, provided the original work, first published in JMIR Pediatrics and Parenting, is properly cited. The complete bibliographic information, a link to the original publication on http://pediatrics.jmir.org, as well as this copyright and license information must be included. 